\title{
Integrated Biosensor System for Monitoring and Visualizing Fish Stress Response
}

\author{
Haiyun $\mathrm{Wu},{ }^{1}$ Masataka Murata, ${ }^{2}$ Haruto Matsumoto, ${ }^{2}$ \\ Hitoshi Ohnuki, ${ }^{3}$ and Hideaki Endo ${ }^{1 *}$ \\ ${ }^{1}$ Department of Ocean Sciences, Tokyo University of Marine Science and Technology, \\ 4-5-7, Konan, Minato-ku, Tokyo 108-8477, Japan \\ ${ }^{2}$ Hokkaido Industrial Technology Center, 379-32, Kikyocho, Hakodate-shi, Hokkaido 041-0801, Japan \\ ${ }^{3}$ Department of Marine Electronics and Mechanical Engineering, Tokyo University of Marine Science \\ and Technology, 2-1-6, Ecchujima, Koto-ku, Tokyo 135-8533, Japan
}

(Received September 28, 2020; accepted November 18, 2020)

Keywords: biosensor, fish, stress, monitoring, visualization

In this study, we introduced an integrated biosensor system that can achieve fish stress monitoring (real-time detection of glucose level as stress indicator) and visualization (a color of green, yellow, or red according to the stress level), realized using a multifunctional LED. The glucose concentration measurement by this system is based on the principle of converting the output current value obtained from the biosensor into a voltage value on an electronic substrate circuit. The stress visualization is based on the principle of switching the lighting color of the LED by setting the switching reference value (Vcomp) on the electronic substrate circuit and comparing Vcomp and the output voltage value (Vout) of the biosensor. First, a glucose standard solution was used to calibrate the electronic substrate circuit by calibrating Vcomp according to changes in Vout. Next, the sensor was attached to the test fish (Oreochromis niloticus) to evaluate the practical use of the system. Then, the concentration of dissolved ammonia in water was changed to 10 and $20 \mathrm{mg} \mathrm{L}^{-1}$ as a stress factor, and the stress response and LED lighting color switch according to the stress response were examined.

\section{Introduction}

The stress response of fish is classified as primary, secondary, or tertiary. ${ }^{(1,2)}$ In the primary response, stress-related hormones such as cortisol and catecholamines (e.g., noradrenaline, adrenaline, dopamine) are secreted. In the secondary response, the stress-related hormones cause physiological and biochemical changes, and activate various mechanisms to increase the blood glucose concentration. In the tertiary response, the individual is considered to be in a state inconsistent with survival, resulting in the suppression of reproduction and growth. ${ }^{(3,4)}$

The blood cortisol concentration is used as a significant indicator of stress for the primary response, and the blood glucose concentration is used as an indicator of stress for the secondary response. In particular, the change in the blood glucose concentration during the secondary

*Corresponding author: e-mail: endo@kaiyodai.ac.jp

https://doi.org/10.18494/SAM.2020.3121 
stress response is characterized by a relatively gradual decrease over several hours after an initial increase. ${ }^{(5,6)}$ Therefore, blood glucose concentration is widely used to measure the overall response to stress. Colorimetric assays using a human clinical test kit are generally applied to measure blood glucose levels in fish. Utilizing colorimetric glucose assays with fish, however, requires procedures such as netting the fish, anesthetizing the fish, and blood sampling while the fish is in air. These procedures themselves are stressors, which makes it difficult to assess the undisturbed stress response of the fish. ${ }^{(7,8)}$

To address this issue, we developed wireless biosensor systems for monitoring the stress response of fish and attempted the real-time monitoring of glucose in freely swimming fish. ${ }^{(9-11)}$ These sensor systems use glucose oxidase (GOx), which is immobilized on the working electrode of a sensor as a molecular recognition element. The GOx oxidizes glucose, decomposing it into gluconolactone and hydrogen peroxide. Applying a voltage of $+650 \mathrm{mV}$ to the working electrode oxidizes the hydrogen peroxide and releases electrons. As the amount of electrons released depends on the amount of glucose decomposed by the enzymatic reaction, the glucose concentration in the fish body can be monitored on the basis of the output current of the sensor. To avoid contamination of the sensor, we used eyeball scleral interstitial fluid (EISF) as the sampling fluid for the sensor because EISF has fewer contaminants than blood, and the glucose concentrations of EISF and blood are strongly correlated. ${ }^{(12)}$ Lastly, to more effectively suppress the influence of the few contaminants existing in EISF, the biocompatibility and durability of the sensor were improved by coating its surface with 2-methacryloyloxyethyl phosphorylcholine (MPC) polymer, which has excellent biocompatibility. ${ }^{(13)}$ The data from the sensor was transmitted to a personal computer (PC) using optical communication technology. We selected optical data transmission because, compared with radio waves and sound waves, light consumes less power and has less attenuation in water. By taking advantage of these features, we designed an optical communication transmitter and receiver and succeeded in monitoring the stress responses of fish using the above sensor. ${ }^{(14)}$

While the biosensors developed in our laboratory have made it possible to monitor the stress responses of freely swimming fish, it is necessary to analyze the obtained stress response values using a PC. Using the transmitted values alone, it is difficult to quickly evaluate a fish's response to a stressor. Therefore, we attempted to visualize the stress response in a manner that can be understood quickly. To this end, we developed a communication/visualization module that can be attached to the fish. ${ }^{(15,16)}$ In addition to the communication light-emitting diode (LED), which transmits the sensor output to the PC, we installed a sensor output visualization LED, which changes color from green to yellow to red as the sensor output increases, which supplies an easy-to-understand signal for evaluating the stress response [(Fig. 1(a)].

Although the current version of the sensor has greatly evolved since the original prototype, some aspects of the sensor should be improved. First, because the data communication LED and the stress visualization LED are independent of each other, two LEDs are attached to the fish [Fig. 1(b)], and as a result, the two light signals are mixed and it is difficult to determine which light is from the stress visualization LED. Moreover, using two LEDs increases the power consumption, which decreases the system operation time and increases the weight of the module, thereby increasing the burden on the fish. Furthermore, it was difficult to calibrate 


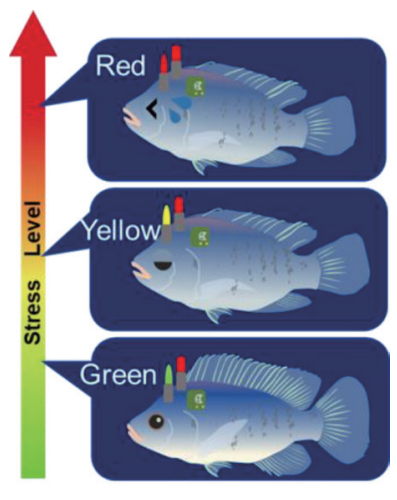

(a)

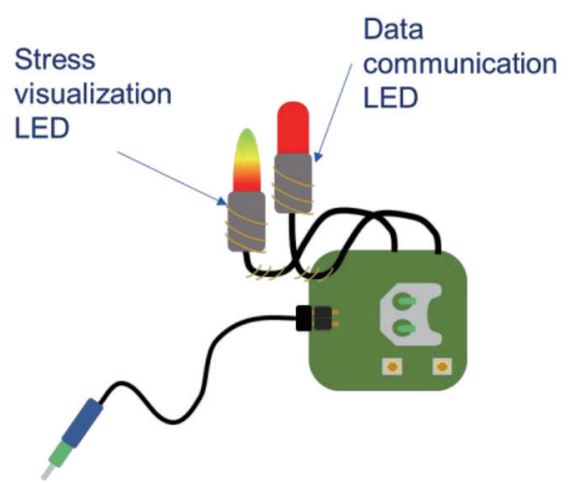

(b)

Fig. 1. (Color online) Schematic of conventional sensor system. (a) Stress visualization system and (b) communication/visualization module.

the LED signal according to the specified degree of stress because the method for setting the reference values for switching the color of the LED was insufficiently studied. ${ }^{(15,16)}$

In the present study, we developed a system that can perform both the communication and visualization functions using a single LED by integrating the conventional data communication LED and stress visualization LED, and a more efficient method for setting the reference values for switching the LED color to realize accurate visualization of the stress response.

\section{Materials and Methods}

\subsection{Reagents}

Glucose oxidase (GOx, 128200 units $^{-1}$ from Aspergillus niger) and bovine serum albumin (BSA) were manufactured by MilliporeSigma (St. Louis, MO, USA). The sodium heparin, Nafion ${ }^{\circledR}$ dispersion solution (DE521 CS type, 5wt\%), 25\% glutaraldehyde solution, glucose kit (glucose CII-Test Wako), 2-phenoxyethanol, and 25\% ammonia water used in this study were manufactured by Wako Pure Chemical Industries, Ltd. (Osaka, Japan). The $\mathrm{D}(+)$-glucose $\left(\mathrm{C}_{6} \mathrm{H}_{12} \mathrm{O}_{6}\right)$, sodium dihydrogen phosphate dihydrate $\left(\mathrm{NaH}_{2} \mathrm{PO}_{4} \cdot 2 \mathrm{H}_{2} \mathrm{O}\right)$, and disodium hydrogen phosphate dodecahydrate $\left(\mathrm{Na}_{2} \mathrm{HPO}_{4} \cdot 12 \mathrm{H}_{2} \mathrm{O}\right)$ used were manufactured by Kokusan Kagaku Co., Ltd. (Tokyo, Japan). All other reagents were reagent grade.

\subsection{Test fish}

Nile tilapia (Oreochromis niloticus) cultivated at the Tokyo University of Marine Science and Technology was used as the test fish. An upper filtration tank (RF-120, Iwaki Co., Ltd., Tokyo, Japan) was installed on a water tank $\left(1200 \times 600 \times 450 \mathrm{~mm}^{3}\right.$, Kotobuki Co., Ltd., Nara, Japan), and aeration was constantly performed with an air pump. The fish were kept at a density of approximately 20 fish per tank. The room temperature was set to $25^{\circ} \mathrm{C}$ and the lighting time was set to 9 h (lights on: 09:00-18:00). 


\subsection{Collection of plasma samples}

A Terumo injection needle $(23 \mathrm{G} \times 1$ ”, $0.60 \times 25 \mathrm{~mm}$, Terumo Co., Tokyo, Japan $)$ was attached to a Terumo syringe $(2.5 \mathrm{~mL})$, which was filled with heparin sodium solution (3000 units $\left.\mathrm{mL}^{-1}\right)$. A test fish was captured with a net and immersed in an anesthetic solution (2-phenoxyethanol, final concentration: $1000 \mu \mathrm{L} \mathrm{L}^{-1}$ in $10 \mathrm{~L}$ of test fish breeding water). After confirming that the fish had reached the stage of paralysis, it was transferred to a worktable, where an injection needle was inserted from the base of the anal fin toward the vertebrae, and blood was drawn from the tail vein. Approximately $0.5 \mathrm{~mL}$ was collected, and the collected blood was dispensed into an Eppendorf tube and immediately centrifuged $(800 \times g, 10 \mathrm{~min}$, $\left.4{ }^{\circ} \mathrm{C}\right)$. The plasma was collected and stored frozen until use.

\subsection{Fabrication of the glucose biosensor}

\subsubsection{Preparation of electrodes}

A biodegradable microelectrode using a platinum-iridium (Pt-Ir) wire as the working electrode and silver-silver chloride $(\mathrm{Ag} / \mathrm{AgCl})$ as the counter electrode was prepared. First, as the working electrode, a Teflon-coated Pt-Ir wire $(\varphi 0.178 \mathrm{~mm}$, Nilaco Co., Ltd., Tokyo, Japan) was cut to a length of approximately $1 \mathrm{~cm}$, and approximately $1 \mathrm{~mm}$ of the Teflon coating on both ends was peeled off using nippers. One of the exposed ends was used as the sensor detection electrode, and a red lead wire ( $\varphi 0.4 \mathrm{~mm}$, Unique Medical Co., Ltd., Tokyo, Japan) was soldered to the other end. Then, approximately $1 \mathrm{~cm}$ of the vinyl coating of a black lead wire ( $\varphi 0.4 \mathrm{~mm}$, Unique Medical Co.) was stripped off, and the exposed copper wire was wrapped around the Teflon coating of the Pt-Ir wire approximately seven times in a spiral. After applying $\mathrm{Ag} / \mathrm{AgCl}$ paste as the counter electrode (BAS Co., Ltd., Tokyo, Japan) to the copper wire wrapped around the Teflon coating, a dry heat sterilizer (Sanyo Electric Co., Ltd., Osaka, Japan) was used to dry the $\mathrm{Ag} / \mathrm{AgCl}$ paste at $90{ }^{\circ} \mathrm{C}$ for $20 \mathrm{~min}$. The $\mathrm{Ag} / \mathrm{AgCl}$ coating and drying operation were repeated. Finally, heat-shrink tubing (Kyowa Harmonet Co., Ltd., Kyoto, Japan) and Araldite adhesive (Huntsman Japan Co., Ltd., Hyogo, Japan) were used to cover the joints of each wire to make them waterproof.

\subsubsection{Immobilization of the enzyme and formation of the biocompatible membrane}

The working electrode of the sensor was immersed in a $5 \%$ Nafion ${ }^{\circledR}$ dispersion solution for $1 \mathrm{~min}$ and dried at room temperature for $10 \mathrm{~min}$ to form a Nafion ${ }^{\circledR}$ membrane. This membrane was used to prevent in vivo contaminants that react at lower potentials than glucose from affecting the sensor's output current. The working electrode was then immersed in the GOx enzyme solution for $1 \mathrm{~min}$ and dried at room temperature for $10 \mathrm{~min}$. This immersion and drying operation was repeated three times. The sensor was then placed in a Petri dish, and $50 \mu \mathrm{L}$ of $25 \%$ glutaraldehyde solution was added dropwise around the sensor, and the Petri dish was sealed with parafilm. Immobilization of the enzyme with glutaraldehyde utilizes Schiff 
base formation, which occurs in weak alkalinity, such as the condensation of glutaraldehyde with lysine residues of GOx and BSA. After leaving the sensor in an incubator at $35^{\circ} \mathrm{C}$ for $6 \mathrm{~h}$, the sensor was immersed in phosphate buffer and kept at $4{ }^{\circ} \mathrm{C}$ in a refrigerator. In addition, before attaching the electrode to the fish body, the sensor was immersed in MPC polymer (Lipidure BL 206, NOF Corporation, Tokyo, Japan) for $10 \mathrm{~s}$ to prevent the adhesion of proteins and cells, etc., and kept at room temperature for $10 \mathrm{~min}$. This operation was repeated three times.

\subsection{LED communication/visualization integrated system}

We developed an LED communication/visualization integrated system (referred to as the LED system) for visualizing the fish stress response (green $\Leftrightarrow$ yellow $\Leftrightarrow$ red) while simultaneously transmitting the sensor output using visible light communication with a single LED on the module attached to the fish body. The LED system changes the LED color from green to yellow and then to red as the stress response of the fish increases.

\subsubsection{Development of the LED system}

The LED system [Fig. 2(a)] comprises a receiver and a data transmission and stress visualization module [Fig. 2(b)] equipped with an integrated LED. The weight of the module is about $3 \mathrm{~g}$ (without battery).

The photoreceiver comprises a photosensor module (H10722-01, Hamamatsu Photonics KK, Shizuoka, Japan). For the module, a circuit was constructed so that a voltage of $+650 \mathrm{mV}$ was applied between the connectors connecting the glucose biosensors. In addition, the circuit converts the current obtained from the glucose biosensor to an output voltage (Vout) using an I/V converter. The controller can adjust the communication sensitivity and signal wavelength range.

Vout was converted from an analogue signal to a digital signal using an A/D converter, processed by a microcomputer, and then transmitted from the module by optical communication using an LED (OSRGHC3131A, OptoSupply Limited, Hong Kong) as the light source. The

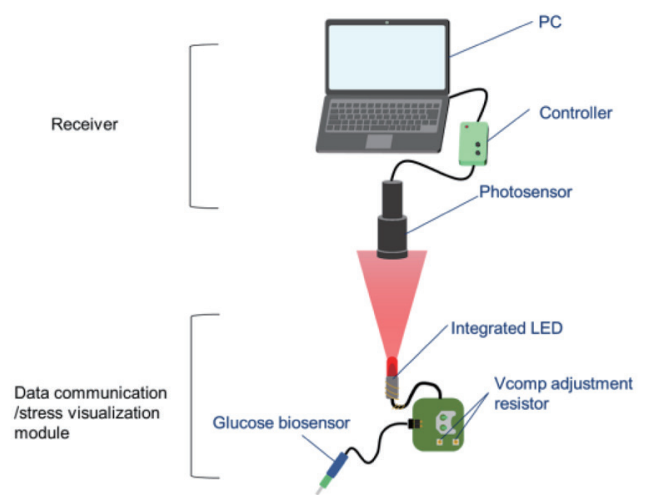

(a)

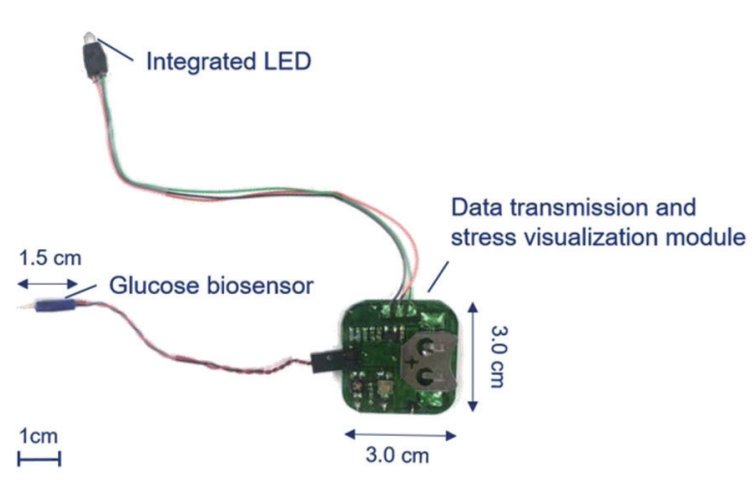

(b)

Fig. 2. (Color online) (a) Schematic of proposed LED system and (b) photo of integrated biosensor. 
optical signal was received by a photosensor, and the output voltage value was converted to the output current value on the PC and output as the measured value.

In addition, to visualize the stress response, Vout was compared with VcompL and VcompH, which are the voltage reference values for switching the LED color between green and yellow and between yellow and red, respectively. The yellow color was created by turning on the red and green outputs at the same time. Thus, the LED color was green for Vout $<V$ compL, yellow for VcompL $<$ Vout $<$ VcompH, and red for VcompH $<$ Vout (Fig. 3). Also, semifixed resistors were connected to $V c o m p L$ and $V c o m p H$ to set their values according to Ohm's law. A digital multimeter (AD-5529, A\&D Co., Ltd., Tokyo, Japan) was used to set the Vcomp levels.

\subsubsection{Vcomp calibration}

As the capacitors and resistors used in the transmission/stress visualization module each have an inherent error range, the entire circuit has an error range. The error affects Vcomp, and an error $(\varepsilon V c o m p)$ occurs between the theoretical value of Vcomp $\left(V_{c o m p} p_{c a l c d}\right)$, which is used in the circuit design to define the threshold voltage for switching the LED color, and the actually measured value ( $\left.V_{c o m p} p_{o b s}\right)$ of Vcomp, which causes the actual switching. To correct the error when setting Vcomp, we examined the VcompL and VcompH errors. When the output current value of the glucose biosensor changes, the Vcomp error also changes. Therefore, the output current value of the glucose biosensor was changed from 2 to $15 \mathrm{nA}$ at $1 \mathrm{nA}$ intervals, and the error of Vcomp for each output current value was measured.

We measured $V c o m p L_{o b s}$ and $V c o m p H_{o b s}$ for each output current value of the glucose

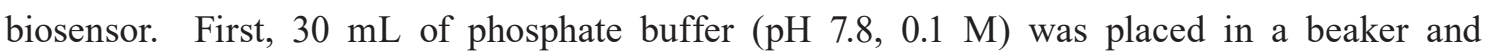
allowed to equilibrate in a $30{ }^{\circ} \mathrm{C}$ thermostatic bath. The glucose biosensor connected to the transmission/stress visualization module was immersed in the phosphate buffer, and the system was turned on. Various amounts of glucose standard solution $\left(5000 \mathrm{mg} \mathrm{dL}^{-1}\right)$ were added to the beaker and the output current was recorded after the glucose biosensor output current stabilized. Vcomp was arbitrarily set for each output current value and the LED color at that time was recorded. The process was repeated 25 times for each output current value, and Vcompobs was obtained.

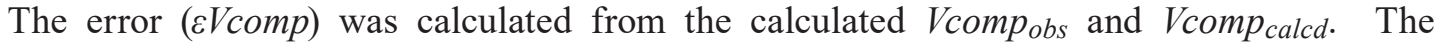
equation for calculating the error is shown below.

$$
\varepsilon V \text { comp }=\text { Vcomp }_{\text {obs }}-\text { Vcomp }_{\text {calcd }}
$$

\begin{aligned} & \hline VcompH $<$ Vout $\longrightarrow$ Red \\ & \hline VcompL $<$ Vout $<$ VcompH $\longrightarrow$ Yellow \\ & \hline Vout $<$ VcompL $\longrightarrow$ Green \\ & \hline\end{aligned}

Fig. 3. (Color online) Relationship between output voltage value and lighting color. Vout: Output voltage value obtained from the sensor; VcompL: Reference value for switching between green and yellow; VcompH: Reference value for switching between yellow and red. 
A simple $3.0 \mathrm{~V}$ constant-current device was used as the power supply for the module. Examining the error of Vcomp confirmed that there was no difference in its value from that of the lithium-ion battery used for the lighting color switching (in vivo), which is described later.

\subsection{Examination of the lighting color switching in vivo}

In this experiment, we attached the LED module to swimming fish and examined the switching of the LED lighting color in vivo. The dissolved ammonia concentration was changed as a stress factor, and the stress response of the test fish was measured by visualizing the switching of the LED lighting color according to the stress response.

\subsubsection{Selection of sample fish and adaptation to the experimental environment}

An experimental water tank $\left(450 \times 300 \times 300 \mathrm{~mm}^{3}\right.$, Marukan Co., Ltd., Osaka, Japan) containing $25 \mathrm{~L}$ of breeding water was prepared. The test fish were arbitrarily selected from the breeding aquarium, transferred to the prepared experimental aquarium, and left in that state for 2 days or more to acclimate. Moreover, to suppress an increase in blood glucose concentration due to feeding, fasting was performed for $12 \mathrm{~h}$ or more before the start of the stress response monitoring. The mean length of the test fish was $29.7 \mathrm{~cm}$ and the mean weight was $334.2 \mathrm{~g}$.

During isolation in the experimental water tank, the ammonia reagent $\mathrm{NH}_{3} / \mathrm{NH}_{4}$ (Spectrum Brands Japan Co., Ltd., Kanagawa, Japan) was dissolved in the breeding water and the ammonia concentration was confirmed.

\subsubsection{Waterproofing the module}

A polyethylene vinyl bag was prepared, heat-compressed using a sealer (Shura Sealer NL101J, manufactured by Ishizaki Denki Seisakusho, Tokyo, Japan), and heat-sealed according to the size of the electronic board of the module. Next, a $2 \mathrm{~mm}$ notch was made at the corner of the polyethylene vinyl bag. A polyethylene hard tube cut to a length of $5 \mathrm{~mm}$ was passed through it. The glucose biosensor lead wire and LED lead wire were removed from the rigid tube, and the electronic substrate part of the transmission/stress visualization module was placed inside the polyethylene vinyl bag, and the glucose biosensor and LED were placed outside the polyethylene vinyl bag. A coating was applied so that the gaps between the polyethylene vinyl bag and the hard tube and between the lead wire and the hard tube were filled with Araldite ${ }^{\circledR}$ adhesive. The module was thereby waterproofed.

\subsubsection{Attaching the module to fish}

The test fish was immersed in anesthesia solution $\left(1000 \mu \mathrm{L} \mathrm{L}^{-1}\right)$, and after confirming that the test fish had reached the paralysis stage, it was transferred to the workbench.

Next, to place the glucose biosensor connected to the module waterproofed as described in Sect. 2.6.2 in the EISF, an injection needle [20G $\times 11 / 2 "(0.90 \times 38 \mathrm{~mm})$, Terumo Co.] was 
inserted into the EISF from the rear of the eye of the test fish, a hole for the EISF was opened, and the glucose biosensor was inserted into the EISF through the hole. A glucose biosensor was fixed to the fish body using an instant adhesive (Aron Alpha, Toagosei Co., Ltd., Tokyo, Japan) as shown in Fig. 4.

Finally, the wire was wrapped around the LED and the lead wire connecting the LED and the electronic board so that the angle could be adjusted, and the LED was attached to the fish body using a tracker (TG-M, Max Co., Tokyo, Japan). The system was operated by a $3.0 \mathrm{~V}$ lithium-ion battery (CR1220, Sanyo Electric Co., Ltd., Osaka, Japan).

\subsubsection{Calibration of glucose biosensor}

The one-point or two-point calibration method is used for the calibration of the output current value in in vivo measurement. Previous research in this laboratory revealed that the one-point calibration method was superior from the viewpoints of the correlation between the glucose conversion value of the sensor and the actual glucose value and the simplicity. ${ }^{(17)}$ Therefore, the one-point calibration method was adopted in this study. The formula of the onepoint calibration method is shown below.

The one-point calibration method was used to obtain the relationship between the output current value and the blood glucose concentration by using a blood sample obtained by a single blood draw. The sensitivity $S\left(\mathrm{nA} / \mathrm{mg} \mathrm{dL}^{-1}\right)$ of the sensor is derived from the glucose concentration $\left(G_{1}\right)$ of the blood sample obtained by blood sampling and the output current value $\left(I_{1}\right)$ of the sensor at that time. The blood glucose concentration $G_{(t)}\left(\mathrm{mg} \mathrm{dL}^{-1}\right)$ can be converted from the sensor sensitivity and the obtained output current value $I_{(t)}(\mathrm{nA})$.

$$
\begin{gathered}
G_{(t)}=I_{(t)} / S \\
S=I_{1} / G_{1}
\end{gathered}
$$

With the transmission/stress visualization module attached to the fish body using the method in Sect. 2.6.3, when the output current value was stable, blood was collected using the method in Sect. 2.3 and plasma samples were collected. The glucose concentration in the collected plasma

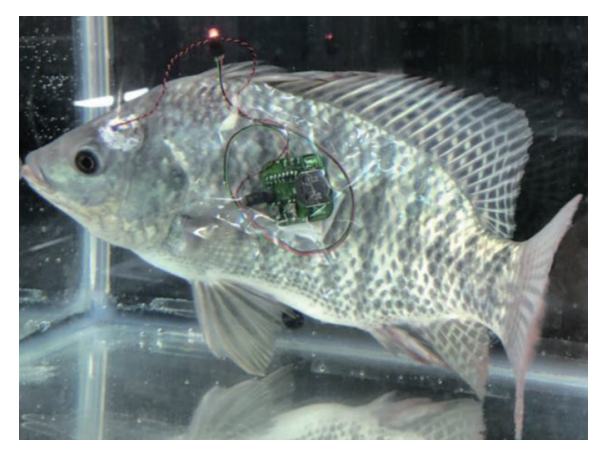

Fig. 4. (Color online) System attachment on fish. 
samples was determined using a glucose kit, and one-point calibration was performed using the value and the output current value at the time of blood sampling.

\subsubsection{Setting of Vcomp value}

To change the lighting color of the LED according to the stress response of the test fish, we set $V$ compL and $V c o m p H$, which were the reference values for switching the lighting color from green to yellow and $V c o m p H$ from yellow to red, respectively.

Considering the normal blood glucose concentration of the test fish obtained in the procedure described in Sect. 2.6.4, VcompL is where the blood glucose concentration becomes the output current value equivalent to $70 \mathrm{mg} \mathrm{dL}^{-1}$, and the output current value corresponding to $90 \mathrm{mg} \mathrm{dL}^{-1}$ was set to $V$ compH. The conversion equations are shown below.

$$
\begin{gathered}
\text { Icomp }=\text { Gcomp } \cdot S \\
V \text { comp } p_{\text {calcd }}=\text { Icomp } \cdot R+\text { Voffset } \\
V \text { comp }=V_{\text {comp }} \text { calcd }+ \text { EVcomp }
\end{gathered}
$$

From the sensor sensitivity $\left[S\left(\mathrm{nA} / \mathrm{mg} \mathrm{dL}^{-1}\right)\right]$ obtained in the one-point calibration method, the blood glucose concentration (Gcomp) set to Vcomp is converted to the current value (Icomp) set to Vcomp. The offset voltage (Voffset) and the resistance value $(R)$ were converted to the voltage value ( Vcomp $\left._{\text {calcd }}\right)$ using Ohm's law. Finally, the error was corrected by adding the calculated error ( $\varepsilon$ Vcomp) to Vcomp calcd to obtain Vcomp.

\subsubsection{Examination of lighting color switching for stress response to ammonia}

A system for monitoring the stress response to ammonia was developed using the method described above, and the test fish was acclimated to the environment for at least $12 \mathrm{~h}$. When the output current value was confirmed to be stable, the stress response monitoring was started.

After calibrating the glucose biosensor and setting Vcomp, it was confirmed that $\mathrm{NH}_{3} / \mathrm{NH}_{4}$ was $0 \mathrm{mg} \mathrm{L}^{-1}$ using a simple ammonia measurement reagent, and the dissolved ammonia concentration in the water tank was increased to $10 \mathrm{mg} \mathrm{L}^{-1}$. The test fish was transferred to the aquarium and subjected to stress due to the increase in ammonia concentration. After $20 \mathrm{~min}$, the test fish was transferred to a tank with a dissolved ammonia concentration of $20 \mathrm{mg} \mathrm{L}^{-1}$, and the switching of the LED lighting color according to the stress response of the test fish was examined.

We also investigated the characteristics of the glucose biosensor in this system. That is, the value measured by the sensor and the actual blood glucose concentration obtained by the colorimetric method using a glucose kit (hereinafter referred to as the conventional method) were compared.

For the conventional method, in this experiment, in addition to the blood collected for the calibration of the biosensor, three further blood collections were performed. First, plasma 
samples were collected approximately $10 \mathrm{~min}$ after the test fish was exposed to the dissolved ammonia concentration of $10 \mathrm{mg} \mathrm{L}^{-1}$, and thereafter at intervals of approximately $20 \mathrm{~min}$. The glucose concentration of each sample was measured by the conventional method, and the stress response monitoring by the communication/visualization integrated system was verified by comparison with the value measured by the sensor.

\section{Results and Discussion}

\subsection{Development of LED communication/visualization integrated system}

The system developed in this study performs both communication and visualization with one LED. Because the number of LEDs has been reduced from two to one, the power consumption of the system is decreased, which increases its total operating time. Furthermore, the weight of the system was reduced from about $6 \mathrm{~g}$ to about $4 \mathrm{~g}$, which is expected to reduce the burden on the fish body.

\subsection{Calibration of LED communication/visualization integrated system module}

In the previous system, the lighting color did not switch according to the value of the set Vcomp. As a countermeasure in that system, the value obtained by subtracting $10 \mathrm{mV}$ from the Vcomp value that theoretically causes switching was set to Vcomp for convenience. Owing to factors such as output current noise and measurement errors caused by electronic components on the board, however, the lighting color often did not switch as expected even when $10 \mathrm{mV}$ was subtracted from the theoretical value [Fig. 5(a)]. Therefore, in the present study, the definition

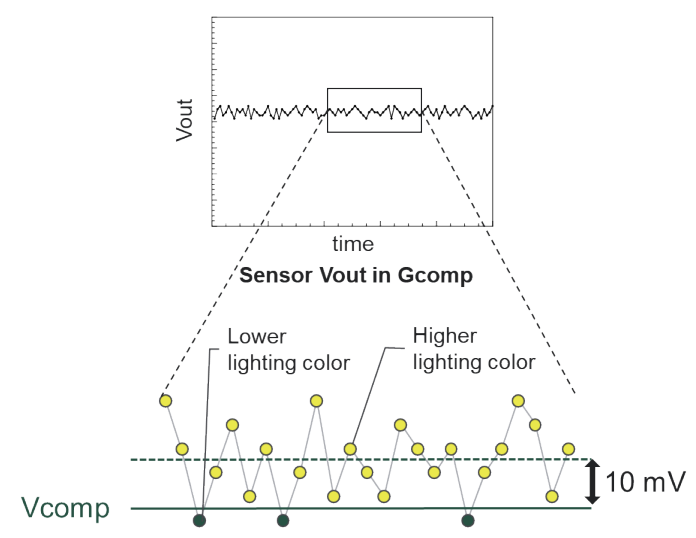

(a)

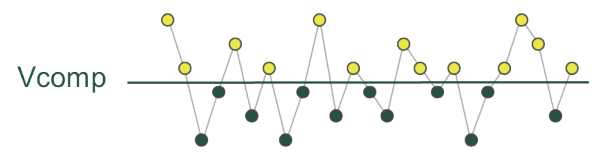

(b)

Fig. 5. (Color online) Calibration of Vcomp setting. (a) Conventional sensor calibrated by subtracting $10 \mathrm{mV}$ from each data. (b) Proposed sensor calibrated by determining the same lighting frequency on both the lower and higher lighting colors. 
of Vcomp was amended so that the lighting frequency of the lighting color was equal to the lighting frequencies of the lower and higher colors [Fig. 5(b)]. The results are shown in Tables 1 (VcompL) and 2 (VcompH).

From these tables, the value for which the ratio of the green lighting color is $50 \%( \pm 10 \%)$ among 25 lightings is $V c o m p L_{o b s}$, and that for which the ratio of the red lighting color is $50 \%( \pm 10 \%)$ is $V c o m p H_{o b s}$. We confirmed that both $V c o m p L$ and $V c o m p H$ had an error of approximately $-20 \mathrm{mV}$ (min: $-15 \mathrm{mV}$, max: $-30 \mathrm{mV}$ ). Furthermore, we confirmed that the error of Vcomp fluctuates with Vout, and that the difference between $V c o m p L$ and $V c o m p H$ varies even at the same output current value. Therefore, it is necessary to correct the error for the required measurement range of glucose and calibrate $V c o m p L$ and $V c o m p H$ independently.

Table 1

Error calibration of $V c o m p L$.

\begin{tabular}{|c|c|c|c|c|c|c|c|}
\hline $\begin{array}{l}\text { Measured } \\
\text { value }(\mathrm{nA})\end{array}$ & $\begin{array}{l}\text { VcompL } L_{\text {calcd }} \\
(\mathrm{mV})\end{array}$ & $\begin{array}{c}\text { Set VcompL } \\
(\mathrm{mV})\end{array}$ & $\begin{array}{l}\text { Green } \\
\text { (Times) }\end{array}$ & $\begin{array}{r}\text { Yellow } \\
(\text { Times })\end{array}$ & $\begin{array}{c}\text { Overall ratio of green } \\
\text { lighting times }(\%)\end{array}$ & $\begin{array}{l}V_{c o m p L_{o b s}} \\
(\mathrm{mV})\end{array}$ & $\begin{array}{l}\text { Error } \\
(\mathrm{mV})\end{array}$ \\
\hline \multirow[t]{3}{*}{ (1) } & \multirow{3}{*}{690} & 660 & 8 & 17 & 32 & \multirow{3}{*}{665} & \multirow{3}{*}{-25} \\
\hline & & 670 & 16 & 9 & 64 & & \\
\hline & & 680 & 17 & 8 & 68 & & \\
\hline \multirow{3}{*}{3} & \multirow{3}{*}{710} & 680 & 8 & 17 & 32 & \multirow{3}{*}{690} & \multirow{3}{*}{-20} \\
\hline & & 690 & 11 & 14 & 44 & & \\
\hline & & 700 & 15 & 10 & 60 & & \\
\hline \multirow{3}{*}{4} & \multirow{3}{*}{730} & 710 & 9 & 16 & 36 & \multirow{3}{*}{715} & \multirow{3}{*}{-15} \\
\hline & & 720 & 16 & 9 & 64 & & \\
\hline & & 730 & 20 & 5 & 80 & & \\
\hline \multirow{3}{*}{5} & \multirow{3}{*}{750} & 720 & 11 & 14 & 44 & \multirow{3}{*}{720} & \multirow{3}{*}{-30} \\
\hline & & 730 & 15 & 10 & 60 & & \\
\hline & & 740 & 19 & 6 & 76 & & \\
\hline \multirow{3}{*}{6} & \multirow{3}{*}{770} & 740 & 8 & 17 & 32 & \multirow{3}{*}{755} & \multirow{3}{*}{-15} \\
\hline & & 750 & 9 & 16 & 36 & & \\
\hline & & 760 & 18 & 7 & 72 & & \\
\hline \multirow{3}{*}{7} & \multirow{3}{*}{790} & 760 & 8 & 17 & 32 & \multirow{3}{*}{770} & \multirow{3}{*}{-20} \\
\hline & & 770 & 12 & 13 & 48 & & \\
\hline & & 780 & 15 & 10 & 60 & & \\
\hline & & 780 & 6 & 19 & 24 & & \\
\hline 8 & 810 & 790 & 11 & 14 & 44 & 790 & -20 \\
\hline & & 800 & 17 & 8 & 68 & & \\
\hline & & 800 & 3 & 22 & 12 & & \\
\hline 9 & 830 & 810 & 11 & 14 & 44 & 815 & -15 \\
\hline & & 820 & 14 & 11 & 56 & & \\
\hline & & 820 & 8 & 17 & 32 & & \\
\hline 10 & 850 & 830 & 13 & 12 & 52 & 830 & -20 \\
\hline & & 840 & 14 & 11 & 56 & & \\
\hline & & 840 & 9 & 16 & 36 & & \\
\hline 11 & 870 & 850 & 13 & 12 & 52 & 850 & -20 \\
\hline & & 860 & 21 & 4 & 84 & & \\
\hline & & 860 & 10 & 15 & 40 & & \\
\hline 12 & 890 & 870 & 12 & 13 & 48 & 875 & -15 \\
\hline & & 880 & 13 & 12 & 52 & & \\
\hline & & 880 & 8 & 17 & 32 & & \\
\hline 13 & 910 & 890 & 12 & 13 & 48 & 890 & -20 \\
\hline & & 900 & 10 & 15 & 40 & & \\
\hline & & 900 & 7 & 18 & 28 & & \\
\hline 14 & 930 & 910 & 13 & 12 & 52 & 910 & -20 \\
\hline & & 920 & 15 & 10 & 60 & & \\
\hline & & 910 & 5 & 20 & 20 & & \\
\hline 15 & 950 & 920 & 12 & 13 & 48 & 920 & -30 \\
\hline & & 930 & 16 & 9 & 64 & & \\
\hline
\end{tabular}


Table 2

Error calibration of $\mathrm{VcompH}$.

\begin{tabular}{|c|c|c|c|c|c|c|c|}
\hline $\begin{array}{l}\text { Measured } \\
\text { value (nA) }\end{array}$ & $\begin{array}{l}\text { VcompH } H_{\text {calcd }} \\
(\mathrm{mV})\end{array}$ & $\begin{array}{l}\text { Set VcompH } \\
(\mathrm{mV})\end{array}$ & $\begin{array}{c}\text { Red } \\
\text { (Times) }\end{array}$ & $\begin{array}{r}\text { Yellow } \\
\text { (Times) }\end{array}$ & $\begin{array}{c}\text { Overall ratio of yellow } \\
\text { lighting times (\%) }\end{array}$ & $\begin{array}{l}\text { VcompH } H_{o b s} \\
(\mathrm{mV})\end{array}$ & $\begin{array}{l}\text { Error } \\
(\mathrm{mV})\end{array}$ \\
\hline \multirow[t]{3}{*}{ (2) } & \multirow{3}{*}{690} & 660 & 16 & 9 & 64 & \multirow{3}{*}{670} & \multirow{3}{*}{-20} \\
\hline & & 670 & 13 & 12 & 52 & & \\
\hline & & 680 & 11 & 14 & 44 & & \\
\hline \multirow{3}{*}{3} & \multirow{3}{*}{710} & 680 & 16 & 9 & 64 & \multirow{3}{*}{690} & \multirow{3}{*}{-20} \\
\hline & & 690 & 11 & 14 & 44 & & \\
\hline & & 700 & 9 & 16 & 36 & & \\
\hline \multirow{3}{*}{4} & \multirow{3}{*}{730} & 700 & 16 & 9 & 64 & \multirow{3}{*}{715} & \multirow{3}{*}{-15} \\
\hline & & 710 & 17 & 8 & 68 & & \\
\hline & & 720 & 9 & 19 & 36 & & \\
\hline \multirow{3}{*}{5} & \multirow{3}{*}{750} & 720 & 18 & 7 & 72 & \multirow{3}{*}{735} & \multirow{3}{*}{-15} \\
\hline & & 730 & 13 & 12 & 52 & & \\
\hline & & 740 & 13 & 12 & 52 & & \\
\hline \multirow{3}{*}{6} & \multirow{3}{*}{770} & 740 & 14 & 11 & 56 & \multirow{3}{*}{750} & \multirow{3}{*}{-20} \\
\hline & & 750 & 13 & 12 & 52 & & \\
\hline & & 760 & 9 & 16 & 36 & & \\
\hline \multirow{3}{*}{7} & \multirow{3}{*}{790} & 760 & 15 & 10 & 60 & \multirow{3}{*}{770} & \multirow{3}{*}{-20} \\
\hline & & 770 & 12 & 13 & 48 & & \\
\hline & & 780 & 10 & 15 & 40 & & \\
\hline & & 780 & 16 & 9 & 64 & & \\
\hline 8 & 810 & 790 & 15 & 10 & 60 & 790 & -20 \\
\hline & & 800 & 9 & 16 & 36 & & \\
\hline & & 800 & 14 & 11 & 56 & & \\
\hline 9 & 830 & 810 & 13 & 12 & 52 & 810 & -20 \\
\hline & & 820 & 6 & 19 & 24 & & \\
\hline & & 820 & 15 & 10 & 60 & & \\
\hline 10 & 850 & 830 & 13 & 12 & 52 & 830 & -20 \\
\hline & & 840 & 10 & 15 & 40 & & \\
\hline & & 840 & 19 & 6 & 76 & & \\
\hline 11 & 870 & 850 & 10 & 15 & 40 & 850 & -20 \\
\hline & & 860 & 9 & 16 & 36 & & \\
\hline & & 860 & 18 & 7 & 72 & & \\
\hline 12 & 890 & 870 & 13 & 12 & 52 & 870 & -20 \\
\hline & & 880 & 10 & 15 & 40 & & \\
\hline & & 880 & 16 & 9 & 64 & & \\
\hline 13 & 910 & 890 & 11 & 14 & 44 & 890 & -20 \\
\hline & & 900 & 10 & 15 & 40 & & \\
\hline & & 900 & 17 & 8 & 68 & & \\
\hline 14 & 930 & 910 & 9 & 16 & 36 & 905 & -25 \\
\hline & & 920 & 8 & 17 & 32 & & \\
\hline & & 920 & 15 & 10 & 60 & & \\
\hline 15 & 950 & 930 & 12 & 13 & 48 & 930 & -20 \\
\hline & & 940 & 9 & 16 & 36 & & \\
\hline
\end{tabular}

\subsection{Evaluation of the lighting color switch in vivo}

The transmission/stress visualization module was attached to the fish and the dissolved ammonia concentration in the breeding water was increased as a stress factor. The stress response of the test fish while swimming was then evaluated from the switch in the LED lighting color on the basis of the blood glucose concentration.

\subsubsection{Glucose biosensor calibration}

The blood glucose concentration of the test fish was $53.5 \mathrm{mg} \mathrm{dL}^{-1}$, the output current of the sensor at the time of blood sampling was $4.81 \mathrm{nA}$, and the sensor sensitivity $(S)$ was $8.99 \times$ $10^{-2} \mathrm{nA} / \mathrm{mg} \mathrm{dL}^{-1}$. 


\subsubsection{Vcomp settings}

In the conventional Vcomp setting method, after dipping the sensor in the collected EISF, the glucose standard solution was added dropwise to the EISF, and the change in the output current value was obtained at the concentration (GcompL, GcompH) at which the lighting changes to the desired color. The previously described method of setting the converted values to VcompL and $V$ compH was used. When stress response monitoring is performed, however, the lighting color was switched at a value different from Gcomp. It was considered that the reason for this was that the EISF used for Vcomp setting was an artificially created "pseudo" sample whose properties differ from those of the in vivo EISF. Therefore, in the present experiment, we applied a new method of setting Vcomp using the blood glucose concentration value converted from the sensor sensitivity, instead of the glucose concentration in EISF in vitro.

When the blood glucose concentration (GcompL) of $V$ compL was $70 \mathrm{mg} \mathrm{dL}^{-1}$, IcompL calculated from the sensor sensitivity was $6.29 \mathrm{nA}$. Similarly, IcompH was $8.09 \mathrm{nA}$ when GcompH was $90 \mathrm{mg} \mathrm{dL}^{-1}$. Furthermore, when these values were converted to voltage values using Eq. (5), the theoretical values of Vcomp and VcompL $L_{\text {calcd }}$ were $776 \mathrm{mV}$ and that of $V$ compH $H_{\text {calcd }}$ was $812 \mathrm{mV}$. Then, the errors of VcompL and $V$ compH at each output current value were corrected using Tables 1 and 2, and $V c o m p L$ was set to $761 \mathrm{mV}$ and $V c o m p H$ was set to $792 \mathrm{mV}$.

On the basis of these results, applying this setting method with calibration increases the accuracy of the Vcomp setting and the lighting color switches in Gcomp as set.

In the conventional setting method, in addition to the setting itself taking approximately $6 \mathrm{~h}$, complicated operations such as resetting before measurement are required. This new setting method was completed in approximately $30 \mathrm{~min}$ and no resetting was required, making this method very accessible.

\subsubsection{Stress response monitoring in ammonia and examination of LED lighting color}

Figure 6 shows the changes over time in the output voltage value and blood glucose concentration determined by the sensor under stress using ammonia. In Fig. $6, \nabla$ indicates the point where the dissolved ammonia concentration in the breeding water was increased to $10 \mathrm{mg} \mathrm{L}^{-1}$ and $\boldsymbol{\nabla}$ indicates the point where it was increased to $20 \mathrm{mg} \mathrm{L}^{-1}$.

From Fig. 6, Vout of the sensor before stress was 738 to $756 \mathrm{mV}$, which corresponds to a blood glucose concentration of 49.5 to $59.0 \mathrm{mg} \mathrm{dL}^{-1}$. Next, when the dissolved ammonia concentration in the breeding water was set to $10 \mathrm{mg} \mathrm{L}^{-1}$ and the measurement was performed for 30 min under this condition, Vout gradually increased for approximately $15 \mathrm{~min}$. At $15 \mathrm{~min}$, Vout peaked at $778 \mathrm{mV}(71.2 \mathrm{mg} \mathrm{dL}-1)$ and then decreased and remained at around 765 to $768 \mathrm{mV}$ (64.5 to $65.8 \mathrm{mg} \mathrm{dL}^{-1}$ ). A high concentration of ammonia induces stress in fish, and the concentration of dissolved ammonia was $10 \mathrm{mg} \mathrm{L}^{-1}$, which induced stress in the test fish. ${ }^{(18-20)}$ The value of Vout at this time was less than that of VcompL, and the lighting color of the LED remained green. Next, the dissolved ammonia concentration was increased to $20 \mathrm{mg} \mathrm{L}^{-1}$, thereby increasing the stress, and monitoring was performed for approximately $50 \mathrm{~min}$. Vout 


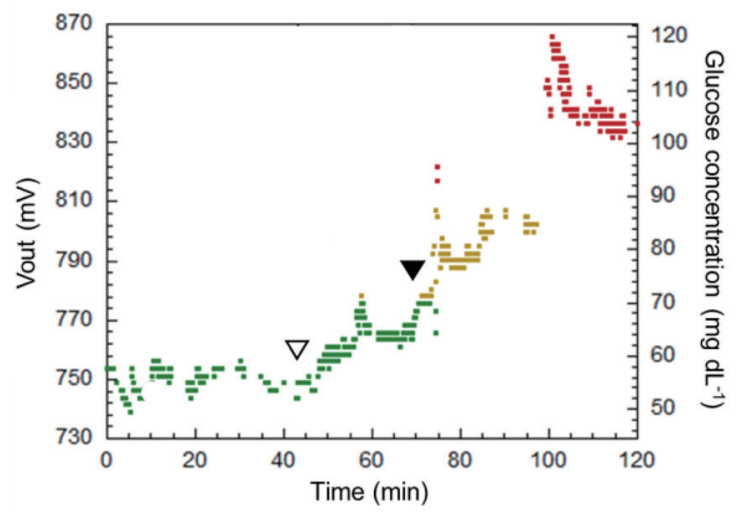

Fig. 6. (Color online) Stress response monitoring in ammonia and examination of LED lighting color. $\nabla$ : Ammonia concentration changed from 0 to $10 \mathrm{mg}$ $\mathrm{L}^{-1}$. $\mathbf{\nabla}$ : Ammonia concentration changed from 10 to $20 \mathrm{mg} \mathrm{L}^{-1}$.

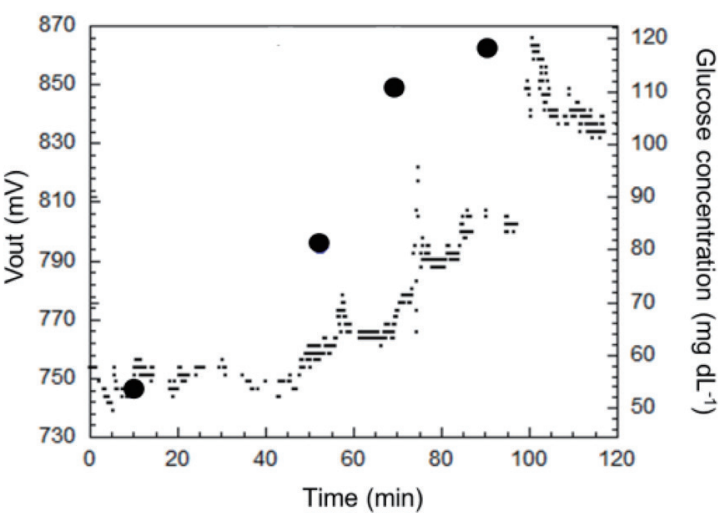

Fig. 7. Correlation between the results of glucose biosensor and conventional method. •: Glucose concentration measured by conventional method.

gradually increased from $768 \mathrm{mV}\left(64.5 \mathrm{mg} \mathrm{dL}{ }^{-1}\right)$ to $807 \mathrm{mV}\left(87.5 \mathrm{mg} \mathrm{dL}^{-1}\right)$ in $20 \mathrm{~min}$ after changing the dissolved ammonia concentration. It is considered that the test fish was subjected to further stress by increasing the dissolved ammonia concentration to $20 \mathrm{mg} \mathrm{L}^{-1}$ (18-20) $^{\text {At this }}$ time, because Vout exceeded VcompL, the LED lighting color changed from green to yellow. After that, Vout decreased and remained at around 800 to $802 \mathrm{mV}\left(83.5\right.$ to $\left.84.8 \mathrm{mg} \mathrm{dL}^{-1}\right)$ before rapidly increasing to $866 \mathrm{mV}\left(120.1 \mathrm{mg} \mathrm{dL}{ }^{-1}\right)$. A high concentration of dissolved ammonia causes fish to swim abnormally, and, in fact, we observed that the test fish swam abnormally around in the aquarium. At this time, the LED lighting color switched from yellow to red because Vout exceeded VcompH. Besides, thanks to the integrated LED, the operating time of the system is theoretically twice that of the conventional system (around $48 \mathrm{~h}$ due to the ratio of light appearance of different colors). Therefore, these findings demonstrated that the stress response of fish can be visually grasped easily and accurately by using this system.

A comparison of the results obtained using the proposed method and the conventional method is shown in Fig. 7. A similar temporal change was observed compared with the conventional method for the glucose concentration measured with the newly improved sensor. Therefore, we confirmed that the integrated LED communication and visualization system can be used to monitor the stress in freely swimming fish in response to changes in the dissolved ammonia concentration in real time.

\section{Conclusion}

In this study, we developed an LED integrated system for data communication/stress response visualization in fish and addressed problems related to the visualization of the stress response encountered using the previously reported system. The findings obtained in this study are summarized below. 
(1) Construction of an LED integrated system for data communication/stress visualization

(a) We succeeded in reducing the weight of the communication/stress visualization module attached to the fish body. (b) Power consumption was also successfully reduced, thus eliminating the need to interrupt the experiment and doubling the time until battery replacement. (c) Because the mixture of light on the communication/stress visualization module was eliminated, the stress response could be visualized more easily.

(2) Method of system setting improved

By correcting the error of Vcomp and changing the setting method of Vcomp and applying the system to test fish, we demonstrated that the lighting color of the LED was more accurately switched at the assumed blood glucose concentration.

The present findings indicate that the proposed system using optical communication technology allows the accurate measurement of the stress response in freely swimming fish in real time and visualization of the stress response, allowing us to determine the degree of stress experienced by the fish. In addition, because this system uses optical communication technology, data can be communicated in an aquatic environment including seawater, also making it applicable to seawater fish. Evaluation of the application of this system was limited to Nile tilapia, however, and further studies should be performed to evaluate the application of this system to other fish species. This system is expected to contribute to the improvement of fish-rearing environments and to facilitate the collection of new knowledge for physiological research fields.

\section{Acknowledgments}

This work was supported in part by a Grant-in-Aid for Scientific Research B (KAKENHI Grant Number 17H03871) from the Ministry of Education, Culture, Sports, Science and Technology of Japan. We wish to thank Dr. Kyoko Hibi (Kokugakuin Tochigi Junior College), Prof. Pierre Morizet-Mahoudeaux (University of Technology of Compiègne, France), and Dr. Chiharu Ichioka (Ichioka Dental Office, Japan) for helpful discussions.

\section{References}

1 S. E. W. Bonga: Physiol. Rev. 77 (1997) 591. https://doi.org/10.1152/physrev.1997.77.3.591

2 B. A. Barton: Integr. Comp. Biol. 42 (2002) 517. https://doi.org/10.1093/icb/42.3.517

3 C. B. Schreck, W. Contreras-Sanchez, and M. S. Fitzpatrick: Reproductive Biotechnology in Finfish Aquaculture, C. Lee and E. M. Donaldson, Eds. (Elsevier Science, Amsterdam, 2001) p. 3. https://doi. org/10.1016/B978-0-444-50913-0.50005-9

4 R. Ashauer, P. Thorbek, J. S. Warinton, J. R. Wheeler, and S. Maund: Environ. Toxicol. Chem. 32 (2013) 954. https://doi.org/10.1002/etc.2144

5 M. Biron and T. J. Benfey: Fish Physiol. Biochem. 13 (1994) 153. https://doi.org/10.1007/BF00004340

6 T. G. Pottinger and T. R. Carrick: Aquaculture 175 (1999) 351. https://doi.org/10.1016/S0044-8486(99)00107-6

7 B. A. Barton: North Am. J. Aquacult. 62 (2000) 12. https://doi.org/10.1577/1548-8454(2000)062<0012:SFDIT $\mathrm{C}>2.0 . \mathrm{CO} ; 2$

8 A. S. Grutter and N. W. Pankhurst: Fish Biol. 57 (2000) 391. https://doi.org/10.1111/j.1095-8649.2000.tb02179.x

9 H. Wu, A. Aoki, T. Arimoto, T. Nakano, H. Ohnuki, M. Murata, H. Ren, and H. Endo: Biosens. Bioelectron. 67 (2015) 503. https://doi.org/10.1016/j.bios.2014.09.015

10 H. Endo and H. Wu: Fish Sci. 85 (2019) 641. https://doi.org/ 10.1007/s12562-019-01318-y 
11 H. Wu, Y. Fujii, T. Nakano, T. Arimoto, M. Murata, H. Matsumoto, Y. Yoshiura, H. Ohnuki, and H. Endo: Sensors 19 (2019) 1518. https://doi.org/ 10.3390/s19071518

12 H. Endo, Y. Yonemori, K. Hibi, H. Ren, T. Hayashi, W. Tsugawa, and K. Sode: Biosens. Bioelectron. 24 (2009) 1417. https://doi.org/10.1016/j.bios.2008.08.038

13 M. Takase, E. Takahashi, M. Murata, H. Ohnuki, K. Hibi, H. Ren, and H. Endo: Int. J. Environ. Anal. Chem. 93 (2013) 125. https://doi.org/10.1080/03067319.2011.649739

14 R. Shinoda, H. Wu, M. Murata, H. Ohnuki, Y. Yoshiura, and H. Endo: Sens. Actuators, B 247 (2017) 765. https://doi.org/10.1016/j.snb.2017.03.034

15 H. Wu, R. Shinoda, M. Murata, H. Matsumoto, H. Ohnuki, and H. Endo: Biosens. Bioelectron. 130 (2019) 360. 10.1016/j.bios.2018.09.042

16 H. Wu, R. Shinoda, M. Murata, H. Matsumoto, H. Ohnuki, and H. Endo: Anal. Methods 11 (2019) 5623. https://doi.org/10.1039/C9AY01752F

17 Z. Mahmoudi, M. D. Johansen, J. S. Christiansen, and O. Hejlesen: J. Diabetes Sci. Technol. 8 (2014) 709. https://doi.org/10.1177/1932296814531356

18 J. W. Meade: Progressive Fish-Culturist 47 (1985) 135. https://doi.org/10.1577/1548-8640(1985)47\%3C135:AA FFC\%3E2.0.CO;2

19 D. J. Randall and A. W. Patricia: Fish Physiol. Biochem. 3 (1987) 107. https://doi.org/10.1007/BF02180412

20 D. J. Randall and T. K. N. Tsui: Mar. Pollut. Bull. 45 (2002) 17. https://doi.org/10.1016/S0025-326X(02)00227-8 